\section{On the Use of Compressed Sensing Techniques for Improving Multistatic Millimeter-Wave Portal-Based Personnel Screening}

Yolanda Rodríguez-Vaqueiro, Student Member, IEEE, Yuri Álvarez, Member, IEEE, Borja Gonzalez-Valdes, Member, IEEE, José Ángel Martínez, Member, IEEE, Fernando LasHeras, Senior Member, IEEE and Carey M. Rappaport, Fellow, IEEE

\begin{abstract}
This work develops Compressed Sensing techniques to improve the performance of an active three dimensional (3D) millimeter wave imaging system for personnel security screening. The system is able to produce a high-resolution 3D reconstruction of the whole human body surface and reveal concealed objects under clothing. Innovative multistatic millimeter wave radar designs and algorithms, which have been previously validated, are combined to improve the reconstruction results over previous approaches. Compressed Sensing techniques are used to drastically reduce the number of sensors, thus simplifying the system design and fabrication. Representative simulation results showing good performance of the proposed system are provided and supported by several sample measurements.
\end{abstract}

Index Terms - Millimeter waves, Imaging Systems, Synthetic Aperture Radar (SAR), Inverse Methods, Fast Multipole Method (FMM), Compressed Sensing.

\section{INTRODUCTION}

$I$ MAGING techniques based on electromagnetic waves are used in a wide range of systems [1]-[5] for security and medical applications. In the area of homeland security, there is an increasing demand for methods to improve the efficiency of the personnel screening for concealed objects and contraband detection at security checkpoints. Human body imaging is an effective way of identifying dangerous objects attached to the body under clothing [3]-[5]. Terahertz wave sensing [6],[7] and X-ray backscatter [8] provide good resolution, but the former is based on expensive, cutting-edge technology and its speed and accuracy depend on precise mechanical scanning, while the X-ray systems make use of ionizing radiation, which is less attractive to the flying public.

Manuscript received November, 2012. This work is supported by CenSSIS, the Gordon Center for Subsurface Sensing and Imaging Systems NSF ERC Program (Award number EEC-9986821); by the Science and Technology Directorate, U.S. Department of Homeland Security under the Award Number "2008-ST-061-ED0001"; by the "Ministerio de Economía y Competitividad" of Spain/FEDER under projects CONSOLIDER-INGENIO CSD2008-00068 (TERASENSE), and TEC2011-24492/TEC (iSCAT).

Y. R. Vaqueiro, B. G. Valdés, J. A. Martínez and C. M. Rappaport are with the ALERT center, Northeastern University, Boston, MA 02115 USA (e-mail: rodriguezvaqueiro.y@husky.neu.edu; bgonzale@ece.neu.edu; jmartine@ece.neu.edu; rappapor@ece.neu.edu).

Y. Álvarez and F. Las-Heras are with the Area of Signal Theory and Communications, Department of Electrical Engineering, Universidad de Oviedo, E-33203, Gijón, SPAIN. Phone: +34-985-182-541; fax: +34-985182-466 (e-mail: yalopez@tsc.uniovi.es ; flasheras@tsc.uniovi.es).
Active nearfield millimeter-wave imaging radar systems are very good candidates for achieving high-resolution imaging, while also balancing the trade-off between accuracy and cost. With mm-wave radar, the object of interest is first illuminated by millimeter waves and then the scattered field is measured and processed in order to reconstruct the surface (or volume) of the object. The image resolution is determined by the radar center frequency, its bandwidth, and its aperture size.

Current state of the art millimeter wave portal imaging systems [3] are based on monostatic radar and Fourier inversion [9]. These systems make use of FFT computation, and thus are inherently fast, but present disadvantages of artifacts when reconstructing dihedrals and corner cubes [10] (such as those used for SAR fiducials) and misrepresenting deeper indentations and protrusions that are incompletely probed with the retro-ray nature of monostatic wave collection. The latter dihedrals effects can be seen in Fig.14 and Fig. 15 in the reference [3], where a high reflectivity value is reconstructed in the void space between the two legs.

This work presents algorithmic improvements to a previous mm-wave based portal system design [11], which is able to generate three-dimensional (3D) high-resolution images of the whole human body using fewer receiving positions. The capabilities of the system are described in the next subsection.

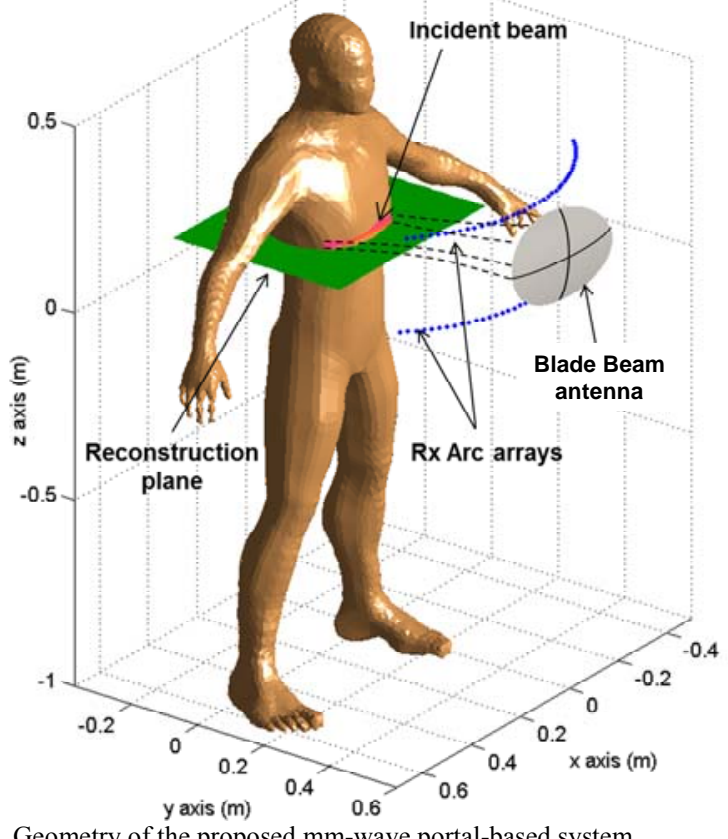

Fig. 1. Geometry of the proposed mm-wave portal-based system.

\section{A. Portal setup}

The general configuration of the proposed system is shown in Fig. 1. The body is illuminated by an incident millimeterwave generated by a novel Blade Beam elliptical-parabolic reflector antenna. This antenna produces a narrow beam in elevation ( $z$ axis) while illuminating the body with constant amplitude in azimuth [12]. This illumination allows thin slices ( 2-3 cm thickness) of the body to be processed independently. The electric field scattered by the section under illumination is captured by two arrays of millimeter wave antenna elements placed on a 90 degree arc above and below the reflector. A SAR based imaging algorithm [13] is used to 
recover the reflectivity values in the z-axis slice illuminated by the Blade Beam antenna. The reflector and receiving antenna arrays are translated vertically, and the two-dimensional retrieved images are stacked to form a full body surface reconstruction. This approach simplifies the three-dimensional reconstruction problem into a combination of multiple $2 \mathrm{D}$ problems which ultimately reduces the computational cost and thus the processing time of the full 3D inversion problem, an essential requirement for practical security screening systems.

\section{B. Aim and scope of this contribution}

One of the limitations of the proposed portal-based system is the minimum number of receivers in the arcs. According to the minimum sampling rate criterion [14], for the proposed system ( $f=60-66 \mathrm{GHz}$, human body having a maximum cross-range size of $\sim 40 \mathrm{~cm}$ ), the minimum number of receivers on each arc to avoid SAR image aliasing is approximately 150 .

Reducing the number of receivers and thereby the cost, complexity, and measurement time without compromising the quality of the reconstructed image, is the purpose of this research. For this goal, Compressed Sensing (CS) techniques, which have been successfully applied in several SAR imaging problems [15]-[19] are proposed.

\section{COMPRESSED SEnsing TECHNIQUE}

Compressed Sensing (CS) [20]-[24] is a relatively novel signal processing theory, which states that sparse signals can be recovered using far fewer samples or measurements than that required by the Nyquist sampling criterion. A discrete signal vector $y \in \mathrm{C}^{\mathrm{N}}$ can be represented in terms of an orthonormal basis $\Psi \in C^{\mathrm{N} \times \mathrm{N}}$ as:

$$
y=\Psi \alpha,
$$

where $\alpha \in C^{N}$ is the coefficients vector. The signal $y$ is $K$ sparse if it is a linear combination of only $K$ basis vectors; that is, $\alpha$ only has $K$ non-zero coefficients, with $K<<N$.

The subsampled signal $y_{s}$, is acquired by linear projection $y_{s}$ $=\Phi y . \Phi \in C^{\mathrm{M} \times \mathrm{N}}$ is a binary matrix: with ones corresponding to the receiver locations, and zeros, to the discarded positions. Selected and discarded positions are randomly selected. $M$ denotes the number of measurements needed, which satisfies $M<<N$.

$$
y_{s}=\Phi y=\Phi \Psi \alpha=\Theta \alpha,
$$

where $\Theta$ is an $M \times N$ matrix. Eq. (2) is underdetermined, that is, it has an infinite set of solutions. Nevertheless, it has been shown that it is very likely to recover $y=\Psi \alpha$ exactly provided that $\alpha$ is a sparse signal and that matrix $\Theta$ obeys a Restricted Isometry Property (RIP), by means of convex programming:

$$
\min _{\alpha \in R^{N}}\|\alpha\|_{1} \text { subject to } y_{s}=\Theta \alpha
$$

When the samples are corrupted with noise, as occurs in real problems, the minimization problem becomes:

$$
\min _{\alpha \in R^{N}}\|\alpha\|_{1} \text { subject to }\left\|y_{s}-\Theta \alpha\right\|_{2}<\varepsilon
$$

Norm-1 and norm-2 are defined as:

$$
\begin{aligned}
& \|\alpha\|_{1}=\sum_{n=1}^{N}\left|\alpha_{n}\right| \\
& \|\alpha\|_{2}=\left(\sum_{n=1}^{N}\left|\alpha_{n}\right|^{2}\right)^{1 / 2}
\end{aligned}
$$

The RIP is satisfied if $\Psi$ and $\Phi$ are incoherent. It has been widely proved [15]-[20] that any random matrix $\Phi$ (as the one described before) is likely incoherent with the orthonormal basis $\Psi$. Numerical evaluation of the coherence (represented by $\left.\mu\left(\Theta^{*}, \Theta\right)\right)$ of $\Theta=\Psi \Phi$, has been assessed as indicated in Eq. (3) of [20]. For the examples shown in this paper, it is verified that $\mu\left(\Theta^{*}, \Theta\right)<\mu\left(\Psi^{*}, \Psi\right)$. A cross-validation technique described in $[21,22]$ is used to determine the value of the regularization parameter $\varepsilon$.

If the CS technique is translated to the SAR imaging problem, $y$ is the entire set of $N$ scattered field measurements, and $y_{s}$, a random set of $M$ measurements given by the linear transformation $y_{s}=\Phi y . \alpha$ is the set of $N$ reflectivity values, $K$ of them being different from zero (or noise-level) values. Orthonormal basis elements are given by:

$$
\begin{aligned}
& \Psi_{m, n}=\exp \left(-j 2 k_{m} R_{m, n}\right) \\
& R_{m, n}=\left(\left(x_{m}-x_{n}{ }^{\prime}\right)^{2}+\left(y_{m}-y_{n}{ }^{\prime}\right)^{2}+\left(z_{m}-z_{n}{ }^{\prime}\right)^{2}\right)^{1 / 2}
\end{aligned}
$$

$M$ is the number of selected frequencies $(F)$ times the number of selected observation points $(P)$. In consequence, wavenumber vector $k$, and scattered field observation points vectors $x, y, z$, are formed as follows:

$$
\begin{aligned}
& M=F \times P \\
& k=\underbrace{[\underbrace{\left[k_{1} \ldots k_{1}\right]}_{P} \underbrace{\left[k_{2} \ldots k_{2}\right] \ldots \underbrace{\left[k_{F} \ldots k_{F}\right]}_{P}]}_{P}}_{F} \\
& x=[\underbrace{\left.\left[x_{1} \ldots x_{P}\right]\left[x_{1} \ldots x_{P}\right] \ldots\left[x_{1} \ldots x_{P}\right]\right]}_{F}
\end{aligned}
$$

In this contribution, Eq. (4) is minimized by means of the Nesterov algorithm [23], provided as a Matlab toolbox. This approach limits the calculation time $(60 \mathrm{~s}$ in a conventional laptop with $N=100 \times 100, M=400$ ). This calculation time can be improved using $\mathrm{C}$ language implementations for a practical real-time system. The choice of this algorithm is based upon superior results when compared to other norm-1 minimization techniques.

The physical basis for the sparsity that is exploited by the CS SAR imaging comes from the fact that the incident field does not penetrate appreciably nor scatter from points inside 
the human body, so for a 2D slice, only a narrow layer, corresponding to the body surface where electric currents are induced, will be recovered. Thus, the resulting 2D image will be sparse.

An additional advantage of CS SAR imaging derived from the aforementioned $\mathrm{mm}$-wave portal-based scanner is that the number of non-zero elements can be estimated using a-priori information. Assuming that the slice where the SAR image is recovered has a size of $(N)^{1 / 2} \times(N)^{1 / 2}$, then it is possible to estimate that the number of non-zero reflectivity elements will be $K \sim(N)^{1 / 2}$ assuming one-pixel range thickness, and that the recovered profile occupies the entire cross-range dimension. Moreover, it has been observed that the sparse signal can be accurately recovered with a number of samples of $M \sim 4 K$ [24]. Thus the expected number of non-zero reflectivity pixels of $\alpha, K$, is related to the minimum number of samples $\left(y_{s}\right.$ vector) $M$ to be used for the reconstruction.

\section{CS HUMAN BODY 3D PROFILE RECONSTRUCTION}

$\mathrm{CS}$ is successful in reducing the amount of samples needed for good inversion results in most SAR imaging systems. Usually, CS images are generated with just $25-30 \%$ of the samples required by the Nyquist sampling criterion. In this section, an application example of the CS techniques for the proposed personnel screening system is presented.

For an initial configuration, a $90^{\circ}$-arc with 150 receiving positions placed at $z=0 \mathrm{~m}$ is considered. As a test case, two metallic objects are placed on top of the human body torso, as shown in Fig. 6 (left).

The forward problem is simulated with physical optics (PO), assuming that the human body behaves as a good conductor in the $60.6-66 \mathrm{GHz}$ frequency band, sampled at 600 $\mathrm{MHz}$ frequency steps $(F=10)$. A Signal-to-Noise ratio of 30 $\mathrm{dB}$ is considered, by adding white Gaussian noise to the simulated scattered field. The reconstruction slice is $(x, y)=(0.3,0.3) \mathrm{m}$, centered at $(x, y)=(0,0.15) \mathrm{m}$, with $z=0 \mathrm{~m}$

First, results for traditional SAR imaging using the algorithm described in [13] are plotted in Fig. 2 (a). Both the human body torso and the objects at the chosen slice are recovered. Next, CS is applied using a subset of $P$ random positions taken from the available 150 samples. Cases for $P=$ 32, 40, and 48 have been tested, finding that $P=40$ provides an acceptable CS profile reconstruction results, providing $90 \%$ probability of correct recovery of the profile. Concerning the coherence assessment, $\mu\left(\Theta^{*}, \Theta\right) \leq 0.35$ for all the tested cases, whereas $\mu\left(\Psi^{*}, \Psi\right)=0.45$. Coherence is normalized so that $\mu \in$ $\left[\begin{array}{ll}0 & 1\end{array}\right]$. For these sample choices, the reconstruction domain has been discretized into $N=80 \times 80,100 \times 100$, and $120 \times 120$ subdomains, to satisfy $M \sim 4 K$, with $K \sim(N)^{1 / 2}$, and $M=F \times P$.

To address the SAR imaging limitations when the Nyquist criterion is not met, results for 40 uniformly spaced and randomly spaced receivers are depicted in Fig. 2 (b) and (c). Aliasing due to cross-range undersampling corrupts the recovered images.

The quality of the reconstructed CS images using 40 receivers (corresponding to $N=100 \times 100$ ) can be clearly appreciated in Fig. 3 when compared to the previous SAR image results. Quantitative assessment of the image quality is provided by the Image Signal-to-Noise Ration (ISNR) defined in Eq. (15) of [22]. Clearly the ISNR of CS images is higher than SAR ones.
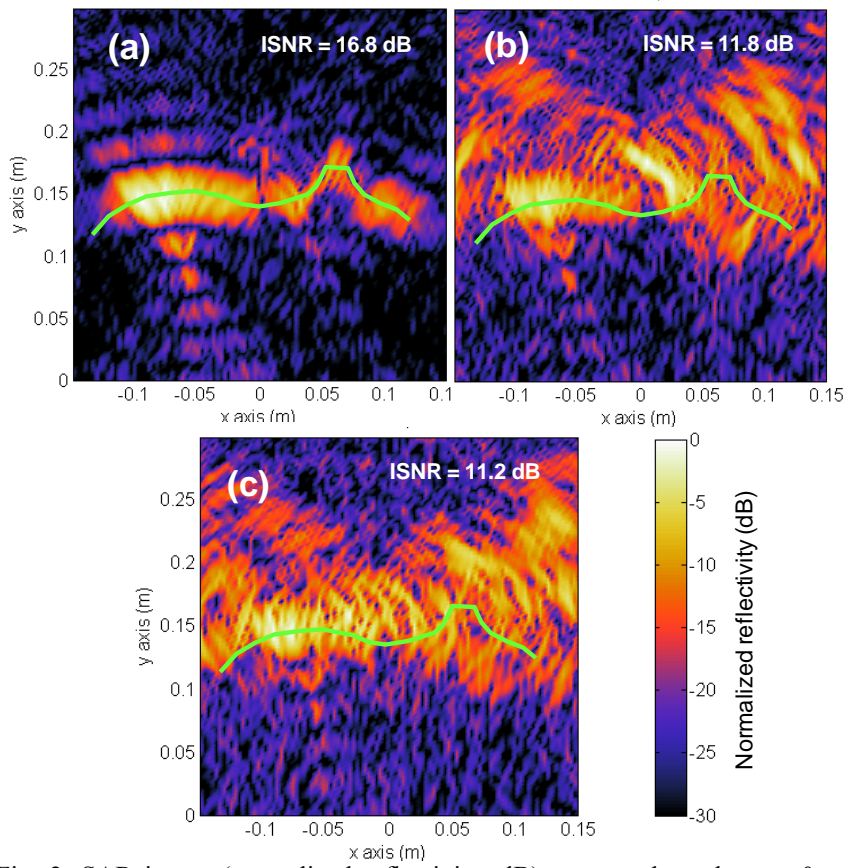

Fig. 2. SAR image (normalized reflectivity, $d B$ ) recovered on the $z=0 \mathrm{~m}$ slice. A single observation arc placed at $\mathrm{z}=0 \mathrm{~m}$ is used. (a) 150 uniformly spaced receivers. (b) 40 uniformly spaced receivers. (c) 40 randomly placed receivers. Solid green line represents the true profile.
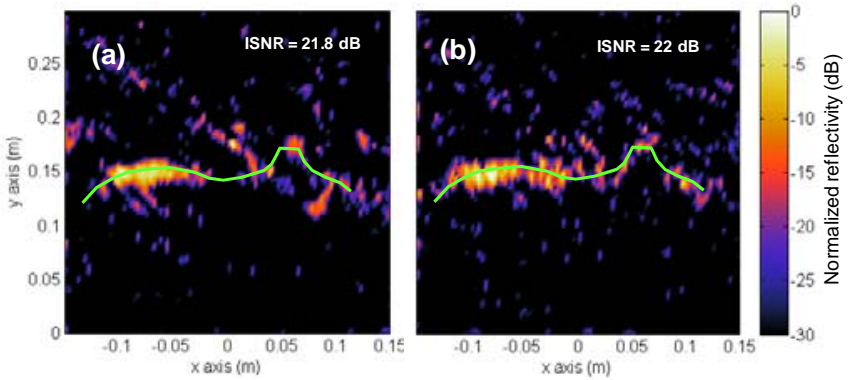

Fig. 3. CS image (normalized reflectivity, $d B$ ) recovered on the $z=0 \mathrm{~m}$ slice. A single observation arc placed at height $z=0 \mathrm{~m}$ is used. (a) 40 uniformly spaced receivers. (b) 40 randomly placed receivers. Solid green line represents the true profile.

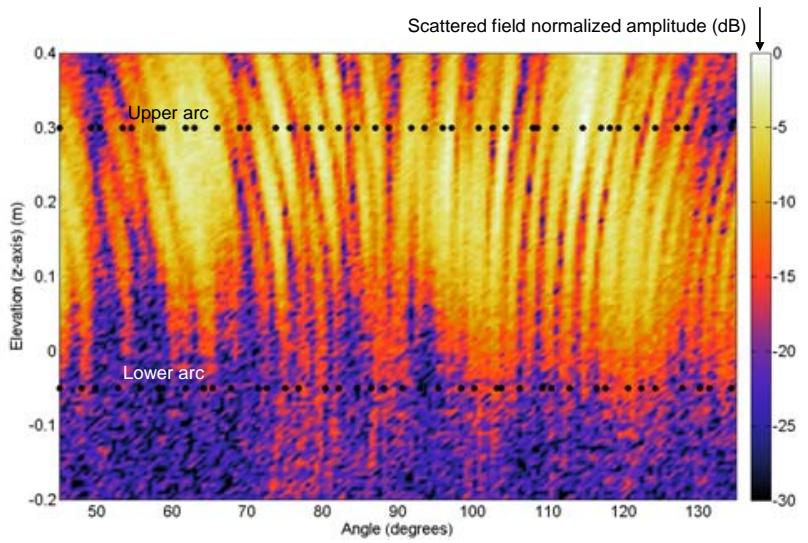

Fig. 4. Field scattered by the human body torso when the beam is pointing at $\mathrm{z}$ $=0 \mathrm{~m}$. Two arcs are used. Black dots indicate the randomly chosen receiver positions. 

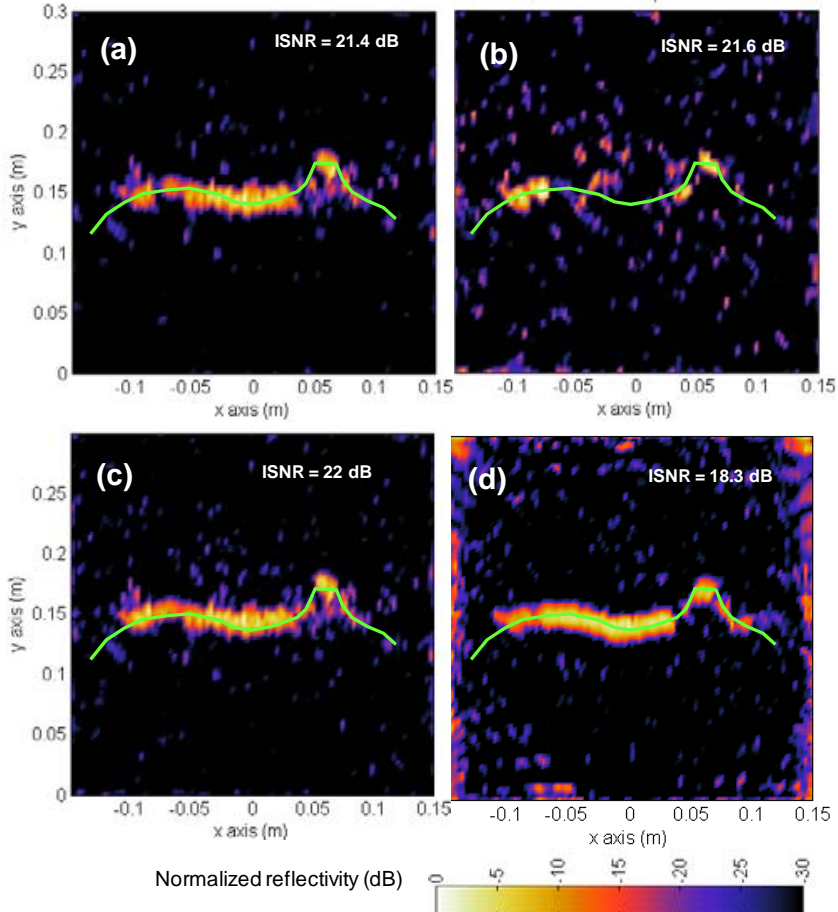

Fig. 5. CS images (normalized reflectivity, $\mathrm{dB}$ ) recovered on the $\mathrm{z}=0 \mathrm{~m}$ slice. (a) CS image using a random set of 40 receivers in the upper arc. (b) CS image using the same random set of 40 receivers in the lower arc. (c) Combination of (a) and (b). (d) CS image using two arcs of 120 equallyspaced receivers. Solid green line represents the true profile.

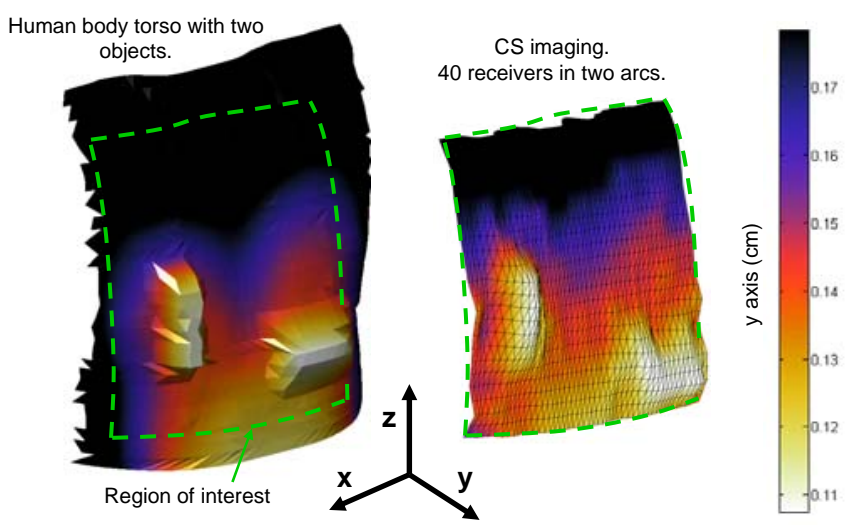

Fig. 6. Original human body torso with two objects mesh (left) and mesh generated from the stacked 2D CS images using two arcs of 40 receivers (right).

In order to confirm that random positions provide better CS results than uniformly spaced receivers, CS image from 40 uniformly spaced receivers (Fig. 3 (a)) is compared to the one provided by 40 randomly selected positions (Fig. 3 (b)). It is observed that the latter fits the true profile better, and also exhibits fewer artifacts.

Once 2D CS capabilities have been proved, next step is the analysis of 3D geometry reconstruction and automatic concealed objects detection using the CS images. As mentioned before, there are few slices in which the human body provides specular reflection in elevation (z-axis). Thus, the use of two arcs, above $(+30 \mathrm{~cm})$ and under $(-5 \mathrm{~cm})$ the blade reflector antenna, ensures that part of the scattered field will be collected in at least one of the two arcs.

The same randomly selected positions are used both in the upper and lower arcs, as shown in Fig. 4. CS images do not really improve when using two different random sets in the arcs. Even more, it has been found that better CS images are achieved if processing every arc independently, then combining the CS images in amplitude and phase.

Fig. 5 represents the CS and SAR images for the case in which the Blade Beam antenna is illuminating the $z=0 \mathrm{~m}$ slice. Figs. 5 (a) and (b) corresponds to the CS images retrieved from the 40 randomly placed receivers placed at upper and lower arcs. As depicted in Fig. 4, the upper arc collects more scattered field power, explaining why the Fig. 5 (a) CS image is better than Fig. 5 (b), as the lower arc collects less signal for a given noise level. Fig. 5 (c) is the incoherent combination of the former ones, slightly improving the quality of Fig. 5 (a). Finally, the CS image using 120 equally-spaced receivers per arc is plotted in Fig. 5 (d) for comparison purposes. It is clear that the CS image can not only be obtained with fewer receivers but also can sharpen the human body profile with respect to SAR (see Fig. 2 (a) and the ISNR provided on each figure), making easier and more accurate automatic profile extraction and 3D mesh generation [11].

The automatic profile extraction described in [11] is applied to CS images to create a 3D mesh, which is depicted in Fig. 6 (right). Twelve slices in $z$ axis, from $-12 \mathrm{~cm}$ to $+10 \mathrm{~cm}$, in 2 cm-steps, are considered. Both the human body torso, as well as the metallic objects placed on it, are accurately retrieved. The Root Mean Square Error (RMSE) on $y$-axis (range or depth) between the original human body torso geometry ( $y_{\text {orig }}$ ) and the retrieved mesh using stacked CS images $\left(y_{C S}\right)$ has been computed (10):

$$
\operatorname{RMSE}=\left(\sum_{n=1}^{N}\left(y_{\text {orig }}-y_{C S}\right)^{2} / N\right)^{1 / 2}
$$

For this case, RMSE $=5.4 \mathrm{~mm}$. Concerning automatic threat detection (which actually is the goal of the mm-wave portal-based system), the generated 3D mesh is smoothed, then subtracting it from the former to obtain a difference mesh. Assuming that the human body torso has smooth variations, objects will show up as protuberances in the difference mesh.

Fig. 7 compares the retrieved difference mesh from stacked 2D SAR images using 150 receivers (Fig. 7 (a)) with stacked 2D CS images with 40 positions (Fig. 7 (b)). In both cases, the metallic objects are clearly identified.

Computational cost is another key factor concerning the final implementation of a real-time portal-based mm-wave imaging system. In this sense, SAR processing is faster than $\mathrm{CS}$, as the former can be easily implemented using forward techniques as described in [13]. For this example, and for every slice, SAR processing using 150 receivers takes $5 \mathrm{~s}$, whereas CS requires $20 \mathrm{~s}$ for the 40 receiver case. A conventional laptop (Intel Core i5 at $2.67 \mathrm{GHz}$ with $4 \mathrm{~GB}$ RAM) has been used for benchmarking. CS has a higher computational cost than SAR, as can be expected when comparing an iterative reconstruction method (CS) with a non- 
iterative method (SAR). An iterative method using SAR as a forward and inverse model will take more time than CS, because the sensing matrix is substantially bigger in the former case.

Despite being 4 times slower, attention must be focused on the overall calculation time for CS: $20 \mathrm{~s}$ times 12 slices $=240$ $\mathrm{s}$, further from the real-time goal. Fortunately, recent advances on Graphics Processing Units (GPUs) applied to inverse problems provide speed-up from 50x to 100x [25].
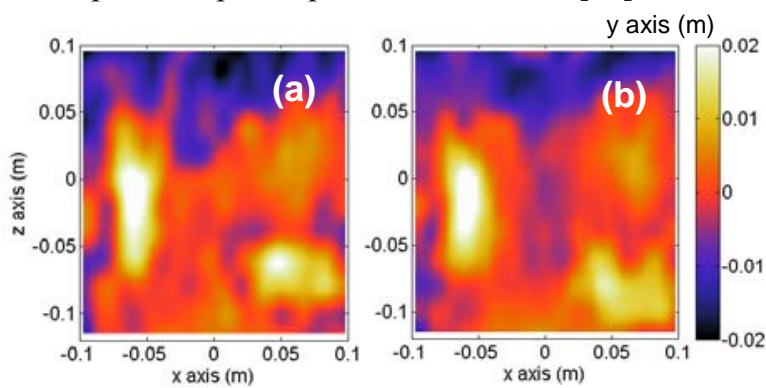

Fig. 7. Detection results. Difference in $y$-axis (range) between the reconstructed $3 \mathrm{D}$ profile and a smoothed one. (a) SAR results using 150 receivers per arc. (b) CS results using 40 receivers per arc.

\section{VAlidation With MEASUREMENTS}

In order to validate the simulation results, a set of experimental measurements were collected. Since the $60 \mathrm{GHz}$ final configuration using the blade beam reflector antenna is still under development and testing, preliminary lower frequency measurements have been performed at the spherical range in anechoic chamber of the University of Oviedo [26]. The measurement setup is depicted in Fig. 8. The objectunder-test (shown in Fig. 8) is a curved metallic surface that aims to model the human body torso curvature. A $2.5 \mathrm{~cm}$ thick metallic object (wood covered with aluminum foil) is placed at the front. The frequency range was 20 to $26 \mathrm{GHz}$, in $F=13$ $500 \mathrm{MHz}$-steps. The field scattered by the metallic target has been measured on a $90^{\circ}$ degrees arc placed $5 \mathrm{~m}$ away of the object. For this configuration, the measurement setup does not allow displacement along $\mathrm{z}$ axis, so the SAR and CS images are recovered in only one slice.

First, the minimum number of samples to avoid SAR image aliasing is calculated. From [14], and assuming that the object has a size of $40 \mathrm{~cm}, 60$ samples are required in the $90^{\circ}$-arc, and as few as 46 equally-spaced samples provide almost aliasing-free image, as depicted in Fig. 9.

Next, CS is applied to a selected random set of positions. Two different sets, with $P=30$ and $P=25$ samples (see Fig. 10 ) are chosen. Thus, as $M=F \times P=390$ and 325 respectively, the reconstruction domain, with size $(x, y)=(0.5,0.5) \mathrm{m}$, is discretized into $N=90 \times 90$ and $N=80 \times 80$ subdomains.

CS and SAR images for the selected set of samples are depicted in Fig. 11. Clearly, SAR images are corrupted by aliasing echoes, thus distorting the proper profile reconstruction. CS overcomes this issue, providing a sharp profile reconstruction (resulting in higher ISNR), requiring as few as 25 samples $(51 \%$ of the information used in the almost aliasing-free Fig. 9 SAR image). For this example, calculation time is $3 \mathrm{~s}$ for the SAR processing with 46 measurement positions and $15 \mathrm{~s}$ for the CS method with 25 positions. The same laptop as in the first example is used.

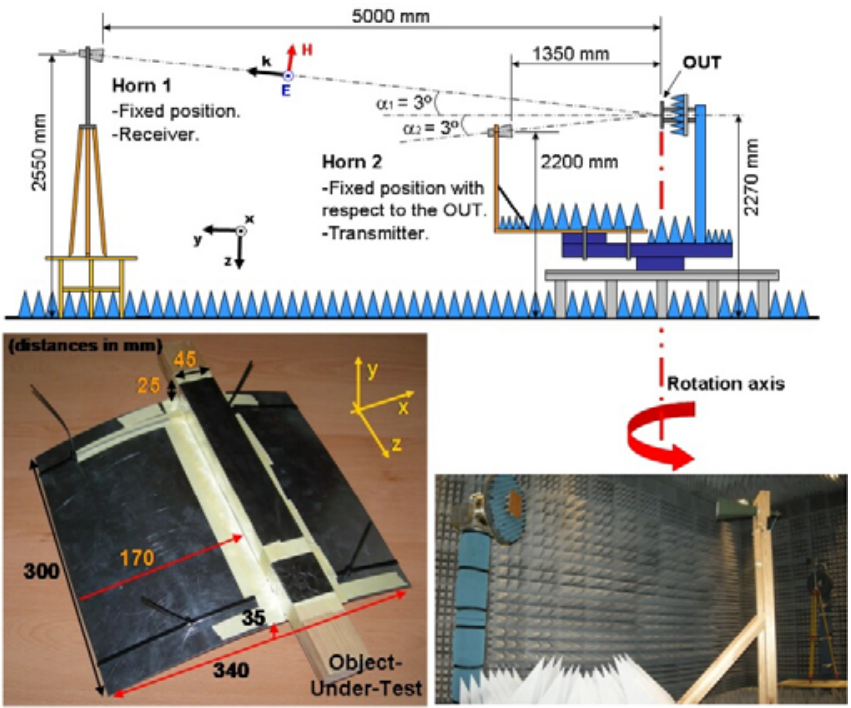

Fig. 8. Experimental measurement setup (top). Metallic curved object-undertest (OUT), which has $z$ axis (height) invariant geometry (bottom left). Anechoic chamber (bottom right).

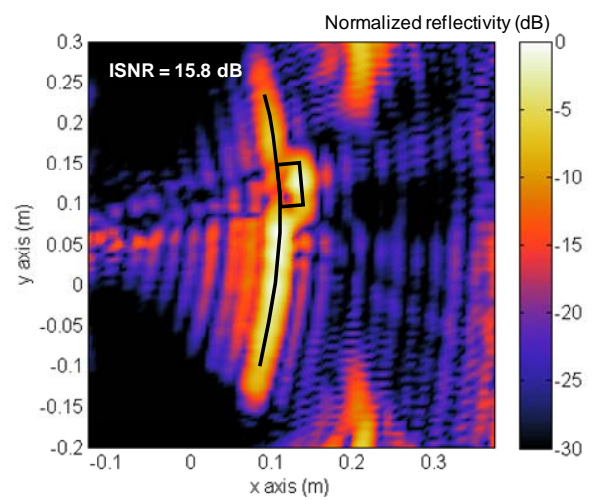

Fig. 9. SAR image (normalized reflectivity, dB) using 46 measurement positions. Aliasing effects are visible along the line $\mathrm{x}=0.2 \mathrm{~m}$. Solid black line represents the true profile.
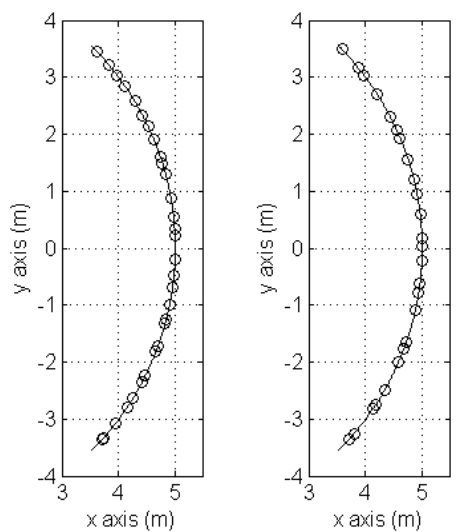

Fig. 10. Selected random measurement positions in the arc. Left: 30 positions. Right: 25 positions.

\section{CONCLUSION}

Compressed Sensing (CS) techniques have been successfully introduced to reduce the number of receivers 
needed for a mm-wave portal-based concealed objects detection system. To achieve similar resolution as SAR imaging, the proposed CS implementation is thus considerable considerably more cost effective (from reducing a 150- to a 40-element arrays) [11], although CS exhibits higher computational time. Results show that CS allows an accurate $3 \mathrm{D}$ profile reconstruction and automatic threat detection using only $25 \%$ of the minimum number of receivers required for traditional SAR imaging processing. Even for an extreme case in which the object under test can be reconstructed with traditional SAR using fewer samples than Nyquist, CS only requires about half of these samples ensuring correct recovery with $>90 \%$ probability.

Concerning mm-wave imaging system implementation, receivers would be permanently mounted at fixed positions in an array. Among several randomly selected positions, some may perform better than others. Thus, further research will be focused on optimizing the receiver positions before final system fabrication.

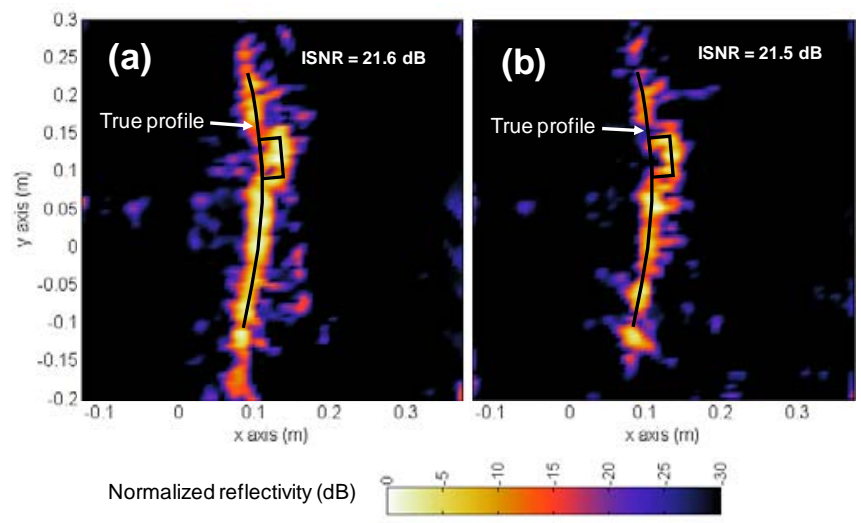

Fig. 11. CS images obtained from 30 (a) and 25 (b) randomly selected positions on the $90^{\circ}$-arc.

\section{REFERENCES}

11] E.C. Fear, X. Li, S.C. Hagness, M. A. Stuchly, "Confocal microwave imaging for breast cancer detection: localization of tumors in three dimensions," IEEE Transactions on Biomedical Engineering, Vol. 49, No. 8, pp 812-822. 2009.

[2] J.A. Martínez-Lorenzo, C. M. Rappaport, F. Quivira, "Physical Limitations on Detecting Tunnels Using Underground-Focusing Spotlight Synthetic Aperture Radar," IEEE Transactions on Geoscience and Remote Sensing, Vol. 49, No. 1, pp. 65-70, Jan. 2011.

[3] D. M. Sheen, D. L. McMakin, T. E. Hall, "Three-Dimensional Millimeter-Wave Imaging for Concealed Weapon Detection," IEEE Transactions on Microwave Theory and Techniques, Vol. 49, No. 9, pp. 1581-1592, September 2001.

[4] J. A. Martínez-Lorenzo, F. Quivira, C. M. Rappaport, "SAR Imaging of Suicide Bombers Wearing Concealed Explosive Threats," Progress In Electromagnetics Research PIER, Vol. 125, pp. 255-272, 2012.

[5] A. Angell, C. Rappaport, "Computational Modeling Analysis of Radar Scattering by Metallic Body-Worn Explosive Devices Covered with Wrinkled Clothing," 2007 IEEE/MTT-S International Microwave Symposium. Honolulu, HI, June 3-8, 2007. pp. 1943-1946.

[6] K. B. Cooper, R. J. Dengler, N. Llombart, B. Thomas, G. Chattopadhyay, P. H. Siegel, "THz Imaging Radar for Standoff Personnel Screening," IEEE Transactions on Terahertz Science and Technology, Vol.1, pp.169-182, Sept. 2011

[7] F. Friederich, W. von Spiegel, M. Bauer, F. Meng, M. Thomson, B. Lisauskas, B. Hils, V. Krozer, A. Keil, T. Löffler, R. Henneberger, A. Huhn, G. Spickermann, P. Bolívar, H. Roskos, “THz Active Imaging
Systems With Real-Time Capabilities", IEEE Transactions on Terahertz Science and Technology, vol. 1, no. 1, pp. 183-200, 2011.

[8] R. F. Eilbert, Shi Shuanghe, "Improved imaging for X-ray inspection systems," IEEE Aerospace and Electronic Systems Magazine, Vol. 20, Issue 3, pp. 23-28, 2005.

[9] J. Lopez-Sanchez and J. Fortuny-Guasch, "3-D radar imaging using range migration techniques," IEEE Transactions on Antennas and Propagation, vol.48, no.5, pp.728-737, May 2000

[10] David K. Barton, Sergey A. Leonov, "Radar Technology Encyclopedia", Artech House, Boston-London, 1998. ISBN 0-89006-893-3.

[11] Yuri Álvarez, Borja Gonzalez-Valdés, José Ángel Martínez, Fernando Las-Heras, Carey M. Rappaport, "3D Whole Body Imaging for Detecting Explosive-Related Threats, “IEEE Transactions on Antennas And Propagation, Vol. 60, No. 9, pp. 4453-4458, September 2012.

[12] C. Rappaport, B. González-Valdés, "The Blade Beam Reflector Antenna for Stacked Nearfield Millimeter-Wave Imaging," IEEE International Symposium on. Antennas and. Propagation, Chicago, July 2012, pp. 1-2.

[13] Y. Alvarez, J. A. Martínez, F. Las-Heras, C. M. Rappaport, "An inverse Fast Multipole Method for geometry reconstruction using scattered field information," IEEE Transactions on Antennas and Propagation, Vol. 60, No. 7, pp. 3351-3360, July 2012.

[14] J. E. Hansen, "Spherical near-field antenna measurements," IEE Electromag. Waves Series 26. London, U.K.: Peter Peregrinus, 1988.

[15] S.-J. Wei, X.-L. Zhang, J. Shi, and G. Xiang, "Sparse Reconstruction for SAR Imaging based on Compressed Sensing," Progress In Electromagnetics Research PIER, Vol. 109, pp. 63-81, 2010.

[16] Vishal M. Patel, Glenn R. Easley, Dennis M. Healy, Jr., Rama Chellappa, "Compressed Sensing with Synthetic Aperture Radar Imaging," Proc. On the 16th IEEE International Conference on Image Processing (ICIP 2009). Cairo, Egypt, 7-10 Nov. 2009. pp. 2141-2144.

[17] Gabriel Rilling, Mike Davies, Bernard Mulgrew, "Compressed sensing based compression of SAR raw data," Proc. On Signal Processing with Adaptive Sparse Structured Representations, SPARS'09. April 06-09, 2009 - Saint-Malo (France). pp. 1-6.

[18] Riafeni Karlina, Motoyuki Sato, "Compressive Sensing for 2D Scan GB_SAR System," Proc. On 1st International Workshop on Compressed Sensing applied to Radar (CoSeRa 2012). 14-16 May 2012. Bonn, Germany. pp. 1-7.

[19] J. A. Martínez-Lorenzo, Y. Rodríguez-Vaqueiro, C. M. Rappaport, A. G. Pino, O. Rubiños, "A compressed sensing approach for detection of explosive threats at standoff distances using a Passive Array of Scatters," In Proc. of IEEE International Conference on Homeland Security Technologies (HST'12). Waltham, MA, November 2012.

[20] J. E. Candès, M. Wakin, "An Introduction to Compressive Sensing," IEEE Signal Processing Magazine, Vol. 25, No. 2, pp. 21-30, March 2008.

[21] A. C. Gurbuz, J. H. McClellan, W. R. Scott, "Compressive sensing for subsurface imaging using ground penetrating radar," Signal Processing, vol. 89, no. 10, pp. 1959-1972, 2009.

[22] Wenji Zhang, Moeness G. Amin, Fauzia Ahmad, Ahmad Hoorfar, and Graeme E. Smith, "Ultrawideband Impulse Radar Through-the-Wall Imaging with Compressive Sensing," International Journal of Antennas and Propagation, vol. 2012, Article ID 251497, 11 pages, 2012.

[23] Stephen Becker, Jérôme Bobin, Emmanuel J. Candès, "NESTA: A Fast and Accurate First-Order Method for Sparse Recovery," SIAM Journal on Imaging Sciences, Vol. 4, No. 1, pp. 1-39. 2011.

[24] A. A. Moghadam, H. Radha, "Complex Sparse Projections for Compressed Sensing," 44th Annual Conference on Information Sciences and Systems (CISS), 2010. 17-19 March, 2010. Princeton, USA, pp. 1-6.

[25] Y. Álvarez, J. Laviada, L. Tirado, C. García, J. A. Martínez, F. LasHeras, C. Rappaport, "Inverse Fast Multipole Method for Monostatic Imaging Applications," IEEE Geoscience and Remote Sensing Letters, Vol. 10, No. 5, pp. 1239-1243, September 2013.

[26] Yuri Alvarez, M. Elena de Cos, and Fernando Las-Heras, "RCS Measurement Setup for Periodic Structure Prototype Characterization," IEEE Antennas and Propagation Magazine. Vol. 52, No. 3, pp. 100-106, June 2010. 